\title{
A Case of Infectious Mononucleosis Complicated by Severe Thrombocytopenia and Hyperuricemia
}

\author{
Vaia Papageorgiou, Georgios Kaltsounis, Konstantinos Loukidis, Anna Kioumi \\ Department of Hematology, Papageorgiou General Hospital of Thessaloniki, Thessaloniki, Greece \\ Email: valiapap@yahoo.com
}

Received 2 November 2015; accepted 15 March 2016; published 18 March 2016

Copyright ( 2016 by authors and Scientific Research Publishing Inc.

This work is licensed under the Creative Commons Attribution International License (CC BY).

http://creativecommons.org/licenses/by/4.0/

(c) (i) Open Access

\begin{abstract}
Infectious mononucleosis (IM) by Epstein-Barr virus is usually a benign, self-limiting disease. Mild to moderate immune thrombocytopenia in the setting of IM is very common and appears in approximately half of these patients, however it rarely presents in severe form and troublesome haemorrhage is even rarer. Rapid spontaneous resolution is possible, with the role of steroids being controversial. Furthermore, elevation of the levels of uric acid because of immune thrombocytopenia is observed in high frequency; however this finding is usually not mentioned. It is attributed to a transient increase in purine turnover, it appears more often in men (with its peak within the first 2 weeks of IM) and it is treated with intravenous fluids and bicarbonates. The present case report describes the case of a young female patient who develops severe thrombocytopenia and intense hyperuricemia following an Epstein-Barr infection. No severe bleeding appeared, levels of uric acid normalised within the first two weeks of treatment and platelet levels rise back to normal within a month under corticosteroid therapy.
\end{abstract}

\section{Keywords}

Infectious Mononucleosis, Thrombocytopenia, Hyperuricemia, Case Report

\section{Introduction}

Infectious mononucleosis (IM) by Epstein-Barr virus is usually a benign, self-limiting disease. Immune thrombocytopenia (IT) in the setting of IM is a well-known entity, but is rarely presented as severe thrombocytopenia. Moreover, elevation of uric acid levels as a consequence of IM is a finding that is not usually mentioned, despite its quite high frequency of appearance. With the present case report, we describe a patient admitted to the hos- 
pital where severe thrombocytopenia (low platelet count) and hyperuricemia (high levels of uric acid in the blood) following an Epstein-Barr infection are revealed.

\section{Case Presentation}

A 20-year-old female Caucasian patient presented in the emergency room, with acute febrile illness lasting for almost two weeks. She referred initially to a local, provincial hospital due to: high fever, myalgia, generalized malaise and sore throat. A diagnosis of Epstein-Barr infection was made based on clinical and laboratory findings [fever, bilateral exudative tonsillitis, splenomegaly, transaminasemia, mild thrombocytopenia, positive heterophile antibody test (mononuclear spot test), lymphocytosis with reactive features on microscopic examination of the peripheral blood smear]. After one day of hospitalization in the provincial hospital, where she was managed with supportive care only, the degree of thrombocytopenia deteriorated (platelet count: $4.000 / \mu \mathrm{L}$ ) but no serious bleeding manifestations were present. However, she was transferred to our tertiary hospital in order to be managed by physicians specialized in hematology. She was an otherwise healthy young adult, carrier of $\beta$ thalassaemia trait.

On admission physical examination revealed: a temperature of $38.5^{\circ} \mathrm{C}$, tonsillar erythyma, exudates and swelling, tachycardia (a pulse rate of 92 per minute at a regular rate), normal blood pressure (110/55 $\mathrm{mmHg}$ ) and a respiratory rate of 16 per minute. Cardiac auscultation revealed no murmurs and no rhonchi or crepitations were found. No serious hemorrhagic diathesis was shown (no gingival bleeding, spontaneous hematomas or petechiae on the palate) apart from localized petechiae of the lower extremities. There was mild conjunctival infection. There was no palpable peripheral lymphadenopathy. The abdomen was soft, her liver not palpable and the spleen was slightly enlarged ( $1 \mathrm{~cm}$ below the left costal margin).

Results of the initial laboratory examination were as follows: Hemoglobin (Hb) $9.9 \mathrm{~g} / \mathrm{dl}$, Hematocrit (Hct) 33.8\%, Mean Corpuscular Volume(MCV) 62.9 fL, Mean Corpuscular Hemoglobin(MCH) 18.4 pg, White Blood Cell count (WBC) 14.170/ $\mu \mathrm{L}$ (Neutrophils 2\%, Lymphocytes 81\%), Platelet count (PLT) 4.000/ $\mu \mathrm{L}$, Serum Glutamic Oxaloacetic Transaminase(SGOT) 91 IU/L, Serum Glutamic-Pyruvic Transaminase (SGPT) 230 IU/L, Alkaline phosphatase $172 \mathrm{IU} / \mathrm{L}$, Uric acid $8.3 \mathrm{mg} / \mathrm{dL}$, Erythrocyte Sedimentation Rate (ESR) $62 \mathrm{~mm}$. Antibodies against Epstein-Barr virus (IgG and IgM) were positive so the diagnosis of IT following Epstein-Barr infection was confirmed.

The patient was treated with intravenous corticosteroids and supportive care (intravenous fluids, bicarbonates, paracetamol) was provided. Temperature fell to normal on the 3rd day of hospitalization. Due to persistent thrombocytopenia (Platelet count: $30.000 / \mu \mathrm{L}$ ) on the 12th day of hospitalization a bone marrow aspirate and biopsy specimen were obtained which showed evidence of peripheral platelet destruction. The transaminases normalized within 11 days of treatment. Her uric acid level had peaked up to $12.4 \mathrm{mg} / \mathrm{dL}$ on the 3rd day but returned to normal values after 11 days of supportive care.

The patient was finally discharged after 16 days of treatment with a stable platelet count of $30.000 / \mu \mathrm{L}$. She continued oral corticosteroid therapy and her platelet count returned within the normal limits after almost a month of corticosteroid therapy. She continued corticosteroids thereafter on a tapering dose. Currently, after almost one and a half year of observation, the patient remains perfectly well with both normal platelet count and uric acid levels.

\section{Discussion}

Infectious mononucleosis is caused by Epstein-Barr virus (EBV). EBV, also called Human Herpesvirus 4 (HHV-4), is a DNA virus, member of the Herpesviridae Family and the Gammaherpesvirinae Subfamily. EBV exhibits tropism for B cells and epithelial cells of the oropharynx and nasopharynx. CD21 surface antigen (also known as CR2) expressed on the surface of those cells acts as the receptor for its major viral envelope glycoprotein, gp350. Fusion and internalization of the virus is triggered by the interaction of a second envelope glycoprotein, gp42, with HLA class II molecules [1].

While mild to moderate decreases in platelet counts -60 to $130 \times 10^{\wedge} 9 / \mathrm{L}$ occur in approximately $50 \%$ of patients with infectious mononucleosis caused by EBV [2], and mild thrombocytopenia is also quite common, severe thrombocytopenia is rare and troublesome haemorrhage is exceedingly rare, occurring in less than $0.05 \%$ of cases [3]. Severe thrombocytopenic episodes may last on occasions for one to several weeks, however rapid spontaneous resolution is possible, as was the case in the presented patient. Steroids are often used in an attempt 
to raise platelet count and prevent dangerous hemorrhage, however their efficacy remains a topic of controversy [3]. In uncomplicated cases of IM, platelet abnormalities such as reduced platelet aggregation and impaired release of platelet factors III and IV are common. It is hypothesized that platelet lesions predisposing to platelet destruction may be implicated in the pathogenetic mechanism of thrombocytopenia [4].

Hyperuricemia appears at a high frequency in patients with infectious mononucleosis (18\% - 66\%), however it is a finding that is often not mentioned [5] [6]. It is a transient finding, with the peak of the levels of serum uric acid occurring within the first 2 weeks of IM, which correlates best with the presence of atypical lymphocytes, a characteristic finding of IM [7]. It appears to develop more often in men than in women. Treatment consists of the administration of intravenous fluids and bicarbonates. No further special treatment is needed. It is believed that in IM, the main cause of hyperuricemia is a transitory increase in purine turnover [8]. Given the correlation between hyperuricemia and rashes caused by ampicillin in the general population, it seems normal that these symptoms often coexist in IM [9].

\section{Conclusion}

Immune thrombocytopenia following Epstein-Barr infection is a well-known clinical entity but is rarely accompanied by severe thrombocytopenia, a consequence that should never be overlooked as it may be life-threatening. Uncomplicated cases of even severe thrombocytopenia may be successfully treated with an excellent prognosis. Uric acid elevation is a usual laboratory finding of IM but is rarely mentioned. To the best of our knowledge there has been no previously reported case of combined severe thrombocytopenia and hyperuricemia associated to Epstein-Barr infection.

\section{Acknowledgements}

Contributions: the authors contributed equally.

\section{Conflict of Interests}

The authors declare no potential conflict of interests.

\section{Consent}

Publication of this case report has been approved by the ethics committee of Papageorgiou Hospital and consent by the patient has been obtained.

\section{References}

[1] Murray, P.R., Rosenthal, K.S. and Pfaller, M.A. (2005) Medical Microbiology. 5th Edition, Elsevier Mosby, Philadelphia.

[2] Carter, R.L. (1965) Platelet Levels in Infectious Mononucleosis. Blood, 25, 817-821.

[3] Wong, S.Y. and Bennett, B. (1982) Natural History of Severe Thrombocytopenia in Infectious Mononucleosis. Postgraduate Medical Journal, 58, 249-251. http://dx.doi.org/10.1136/pgmj.58.678.249

[4] Clancy, R., Jenkins, E. and Firkin, B. (1971) Platelet Defect of Infectious Mononucleosis. British Medical Journal, 4, 646-648. http://dx.doi.org/10.1136/bmj.4.5788.646

[5] Schooley, R.T. and Dolin, R. (1985) Epstein-Barr Virus (Infectious Mononucleosis). In: Mandell, G.L., Douglas, R.G. and Bennett, J.E., Eds, Principles and Practice of Infectious Diseases, John Wiley \& Sons Inc., New York, 971-982

[6] Niederman, J.C. (1983) Infectious Mononucleosis. In: Hoeprich, P.D., Ed, Infectious Diseases, 3rd Edition, Har-Row, Philadelphia, 1205-1211.

[7] Cowdrey, S.C. (1966) Hyperuricemia in Infectious Mononucleosis. JAMA, 196, 107-109. http://dx.doi.org/10.1001/jama.1966.03100170061018

[8] Nessan, V.J., Geerken, R.C. and Ulvilla, J. (1974) Uric Acid Excretion in Infectious Mononucleosis: A Function of Increased Purine Turnover. Journal of Clinical Endocrinology \& Metabolism, 38, 652-654. http://dx.doi.org/10.1210/jcem-38-4-652

[9] Jick, H. and Porter, J.B. (1981) Potentiation of Ampicillin Skin Reactions by Allopurinol or Hyperuricemia. The Journal of Clinical Pharmacology, 21, 456-458. http://dx.doi.org/10.1002/j.1552-4604.1981.tb01749.x 